\title{
ISLA PRÄGT AUCH DIE GESCHICHTE DER LASERAKUPUNKTUR
}

\section{Liebe Kolleginnen und Kollegen,}

die Laserakupunktur ist eine relativ auf das Alter bezogen junge, bereits etablierte Methode auf dem Gebiet der Akupunktur, die seit den frühen 1970er-Jahren erfolgreich zur Behandlung verschiedener Erkrankungen eingesetzt wird. Sie hat trotz ihres ca. 50-jährigen Bestehens bereits eine umfassende Geschichte hinter sich. Ein von einemVerfasser dieses Vorworts in dem Journal Medical Acupuncture (Band 32, Nr. 4) mit dem Titel „History of Laser Acupuncture:A Narrative Review of Scientific Literature“ publizierter Rückblick beschäftigt sich mit Fakten dieser Geschichte und fasst die wichtigsten Meilensteine bis zum Jahr 2020 zusammen. Wissenschaftliche Artikel aus den Datenbanken PubMed, Google Scholar und China National Knowledge Infrastructure (CNKI) dienten als Forschungsdokumente. Darüber hinaus umfasste die Forschung zur Geschichte der Laserakupunktur Material, welches in wissenschaftlichen Büchern veröffentlicht wurde. Insgesamt wurden 38 gelistete Artikel identifiziert, von denen 7 als relevant erachtet wurden. Neben einer allgemein anerkannten Definition der Laserakupunktur, den Anfängen der Laserakupunktur und den ersten klinischen Anwendungen weltweit zeigt die Übersichtsarbeit auch die wichtigsten Meilensteine in der Entwicklung dieser Disziplin auf. Letztere sind natürlich die Entwicklung von Laserakupunktursystemen, erste klinische Studien, die so wichtige Implementierung von Mehrkanal-Laserakupunk- turgeräten und letztlich die Kombination der Laserakupunktur mit Methoden aus der Forschung zur Photobiomodulation. Laserakupunktur kann somit durchaus als ganz essenzieller Teil der Photomedizin generell gesehen werden.

Obwohl bei der Evaluation dieser innovativen Methode der Laserakupunktur erhebliche Fortschritte erzielt wurden, besteht in den kommenden Jahren und Jahrzehnten weiterer Forschungsbedarf. DieVertreter und dankenswerterweise die zahlreichen Mitglieder der ISLAtranscontinental sind trotz COVID-Krise dazu bereit, diese neuen Herausforderungen anzunehmen.

Die ISLA hat mit ihrem 20. Jubiläumskongress, der zu Beginn des Monats September 2021 in Präsenz und online organisiert wurde, die Fortsetzung einer bewährten wissenschaftlichen und praktischen Veranstaltungsserie verwirklicht. Das Weltakupunkturforum in Davos im März 2022 wird - sofern die COVID-Situation es zulässt selbstverständlich den wissenschaftlichen und praktischen Fokus auf die Akupunktur legen, die Laseranwendungen werden dabei aber nicht zu kurz kommen.

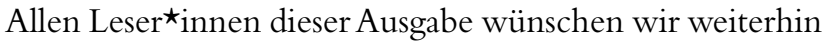
Gesundheit und alles Gute!

M. Weber

G. Litscher

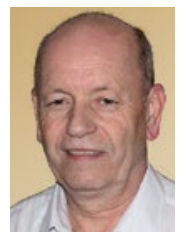

Dr. med. Dipl. Chem. Michael Weber

President for Medical and Clinical Applications der International Society for Medical Laser Applications (ISLAtranscontinental)

Sohnreystrasse 4

37697 Lauenförde

Internet: www.isla-laser.org

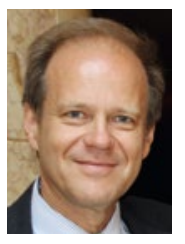

Univ.-Prof. Prof. h.c. DI Dr. techn.

Dr. scient. med. Gerhard Litscher

President for Science and Research der International Society for Medical Laser Applications (ISLAtranscontinental) Medizinische Universität Graz, Auenbruggerplatz 39, EG19, A-8036 Graz, Tel: +43 316/385-83907 E-Mail:gerhard.litscher@medunigraz.at 